\title{
High-order sampling technique for geodetic VLBI and the future
}

\section{Kazuhiro Takefuji*}

National Institute of Information and Communications Technology, 893-1 Hirai, Kashima,

Ibaraki 314-8501, Japan

E-mail: takefujienict.go.jp

Radio Frequency (RF) direct sampling is a technique to sample RF signals directly without the use of a frequency converter and an anti-aliasing filter. In case of geodetic VLBI, RF frequency is at most $9 \mathrm{GHz}$ now. Recently a digital sampler with a high sensitivity at RF frequency higher than $10 \mathrm{GHz}$ has been developed. The sampler enables us to evaluate the use of RF direct sampling technique in geodetic VLBI. RF direct sampling system has a potential to make system simple and stable, because analogue frequency converters are not used unlike conventional system. We have developed RF direct sampling VLBI system and operated them on the Kashima-Tsukuba baseline (about $50 \mathrm{~km}$ in length) in Japan. At first, we carried out VLBI experiment only for $\mathrm{X}$-band $(8 \mathrm{GHz})$ signals. The signals would be sampled directly with under-sampling technique and successfully got the first fringes. Aliased signals could be discriminated through a correlation processing. Then we adopt the RF direct sampling to mix signals, i.e., S-band (2 GHz) and Xband signals are combined each other, so as to make a geodetic VLBI observation. We carried out a 24-hour geodetic VLBI session in 2011 and got consistent results with those obtained by the conventional VLBI. Now we have been developing the next RF direct sampling VLBI system, Gala-V, which is semi compliant with the VLBI2010 specification.

11th European VLBI Network Symposium I\& Users Meeting,

October 9-12, 2012

Bordeaux, France

\footnotetext{
* Speaker.
} 


\section{Introduction}

If analogue electronic is replaced with digital electronic, a local group delay variations caused by temperature could be reduced with benefit of digital data transfer. Also it will reduce the cost of the geodetic VLBI system associated with converters and will increase the reliability of the system. Recently it becomes available to sample RF signals directly due to the progress of a sampling device performance. The ADX-831 which is developed by the ELECS INDUSTRY CO. LTD., is a A/D sampler that has an input bandwidth up to $24 \mathrm{GHz}^{1}$. We temporarily installed the ADX-831 to the Kashima $11 \mathrm{~m}$ station ${ }^{2}$ and Tsukuba $32 \mathrm{~m}^{\text {station }}{ }^{3}$. In this report, we propose a new type of VLBI system with the RF-direct-sampling technique. We report two experimental results. In May 2011 we conducted the first test X-band VLBI experiment with the RF direct sampling technique. In October 2011 we conducted a 24-hour dual-band geodetic VLBI experiment using this technique [四]. We will also report results about the next VLBI system applied for RF direct sampling, which is semi-compliant with the VLBI2010 specifications.

\section{A first fringe test with RF direct sampling system}

The RF-direct-sampling means that directly sampling RF frequency without any frequency conversions is now possible as suggested by its name. We firstly installed this RF direct sampling system to X-band of the Kashima $11 \mathrm{~m}$ antenna and Tsukuba $32 \mathrm{~m}$ antenna to detect the first fringe. The RF signal of the X-band LNA output was transferred to the observation room, then it was directly input to the digital sampler. The ADX-831 digitizes an IF signal with sampling rate at $8192 \mathrm{MHz}$ with 3-bits quantization internally. The digital sampler ADX831 has an analog input range of up to $24 \mathrm{GHz}$, thus it has sufficient sensitivity to process X-band (8-9 GHz) signals (see some specifications in Table.(W). Since the limitations of the recording system were 3 Gbps to 4 Gbps (RAID-6 on sixteen 2 tera byte disks), the sampled data was decimated by an internal FPGA at $1024 \mathrm{MHz}$ with 2-bit quantization in the experiment.

\begin{tabular}{|l|l|}
\hline Frequency range of analog input & $10 \mathrm{MHz}$ to $24 \mathrm{GHz}$ \\
Number of analog input ports & 1 port (in case of $8192 \mathrm{Msps}$ ) or 2 ports (4096 Msps) \\
Sampling rate & $8192 \mathrm{Msps}, 4096 \mathrm{Msps}, 2048 \mathrm{Msps}$, and 1024 Msps \\
Quantization & 3 bits, 2 bits, and 1bit \\
10GbE output & $10 \mathrm{GBASE}-\mathrm{SR}, 10 \mathrm{GBASE}-\mathrm{LR}$, or 10GBASE-ER \\
$10 \mathrm{GbE}$ protocol type & VDIF / UDP / IP \\
\hline
\end{tabular}

Table 1: Specfications of digital A/D sampler ADX831

According to the Nyquist sampling theorem, double sampling rate (i.e., $18 \mathrm{GHz}$ ) is needed to process the full bandwidth of $9 \mathrm{GHz}$ without aliasing. However, if we allow aliasing, we can adopt much lower sampling rate. This technique is called an under sampling or a high-order sampling.

\footnotetext{
${ }^{1}$ http://www.elecs.co.jp/ElecsIndustry/Product/HighspeedOperate/HighspeedADC.html

${ }^{2}$ http://www2.nict.go.jp/aeri/sts/stmg/index_e.html

${ }^{3}$ http://www.spacegeodesy.go.jp/vlbi/en/index.html
} 
If the bandwidth of the signal is less than half of the sampling rate, i.e., $512 \mathrm{MHz}$, and integer multiple of the sampling rate locates at an either edge of the frequency band, the signal can be sampled without any overlap of frequency range after sampling. If we adopt a $1024 \mathrm{MHz}$ sampling speed to this band-limited signal, we can obtain a $512 \mathrm{MHz}$ bandwidth signal which is a half of the sampling speed based on the Nyquist sampling theorem. The relation between spectra after and before sampling in the case of higher order sampling is expressed as,

$$
\begin{array}{r}
P(f)=P(n * f s+f), \quad(0<=f<1 / 2 f s) \\
P(f s-f)=P((n-1) * f s+f), \quad(1 / 2 f s<f<=f s)
\end{array}
$$

where $f$ is the frequency and $f s$ is the sampling rate, and $n=1,2,3, \ldots$. Eq.2.2 is equivalent to

$$
P(f)=P(n * f s-f),(0<=f<1 / 2 f s)
$$

A band given by Eq.2. is called Upper Side Band (USB) and that by Eq. 2.3 an Lower Side Band (LSB). The band-limited X-band signal of $8192 \mathrm{MHz}$ to $8704 \mathrm{MHz}$ becomes USB by 1024 MHz sampling as high-order-sampling.

We observed the radio quasar 3C84 with Kashima $11 \mathrm{~m}$ and Tsukuba $32 \mathrm{~m}$, and recorded the signal with $1024 \mathrm{MHz}$ sampling speed and 2-bits quantization. After the correlation with GICO3, which is a fast software correlator developed by NICT[[]], we could detect the first fringe at X-band with the RF direct sampling system (see Figure.W).
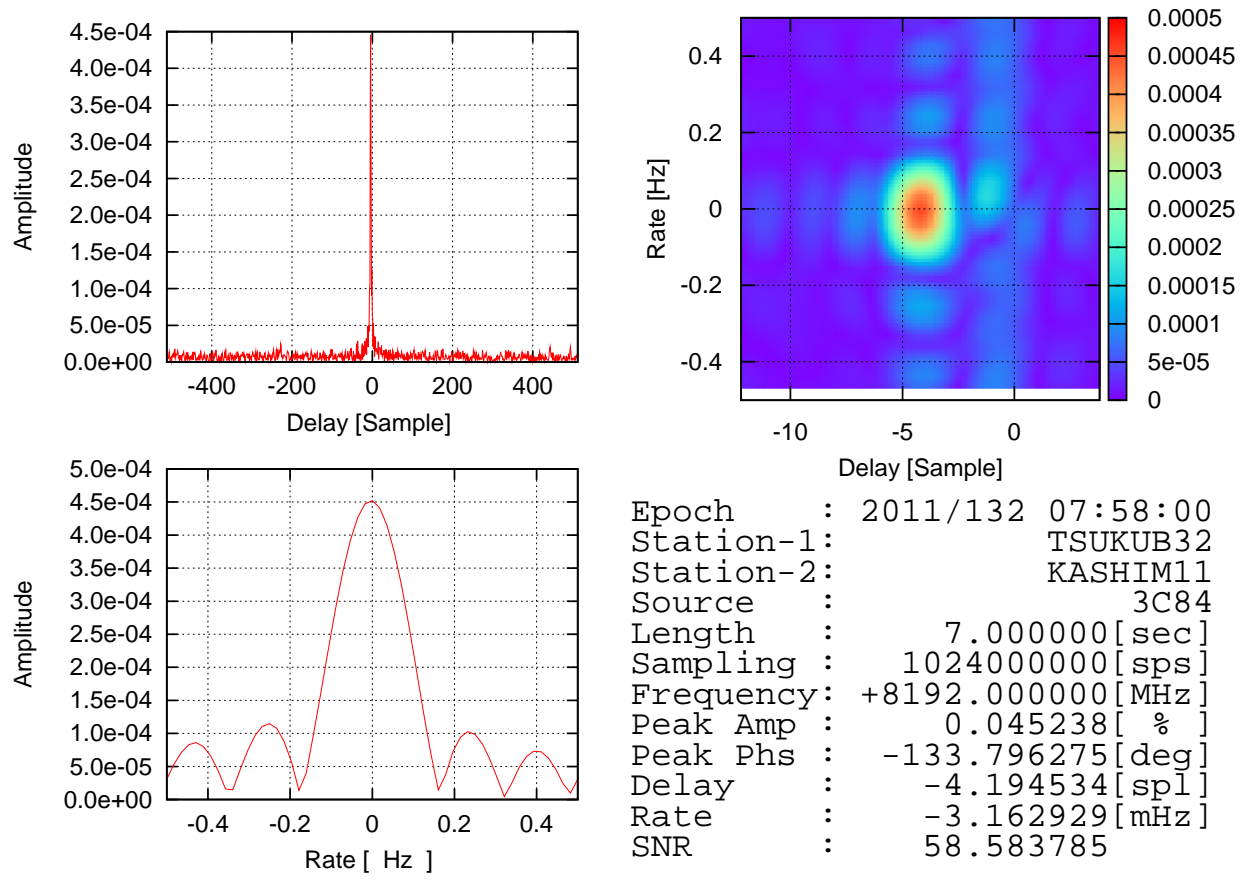

Figure 1: First fringe at $\mathrm{X}$-band with direct sampling system between the Kashima $11 \mathrm{~m}$ antenna and the Tsukuba $32 \mathrm{~m}$ antenna. 


\section{RF direct sampling system applied to the geodetic VLBI}

After we successfully obtained fringes with the RF direct sampling VLBI, we carried out a 24-hour geodetic VLBI using the direct sampling system. The figure.[ shows the diagram of the both systems the Kashima $11 \mathrm{~m}$ and the Tsukuba $32 \mathrm{~m}$ stations. And the figure.[3] shows the spectrum of the combined signal in the frequency range from $\mathrm{DC}$ to $10 \mathrm{GHz}$, which can be seen the combined signals of the S-band and the X-band.

For 24 hours, we observed several times a variety of radio sources. Each scan last 30 seconds, and the number of total scans was 945. During the observation, we recorded the combined RF signal at a 1024 $\mathrm{MHz}$ sampling rate with 2-bits quantization and the total data size became about 7.3 tera bytes at one station. After correlation for the data of the two stations with GICO3, we could detect stable fringes from the S-band and from three $\mathrm{X}$-bands from the single sampled digital data, which includes aliased signals of the X-band and $\mathrm{S}$-band. Thus the sampled data is needed for four times correlations.

Then we performed the bandwidth synthesis for the three X-bands after correlation using the software
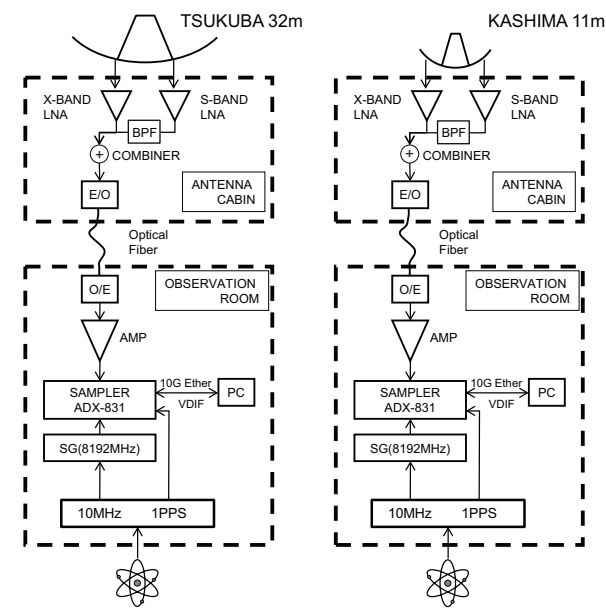

Figure 2: Schematic diagrams of direct sampling VLBI at the Kashima $11 \mathrm{~m}$ and Tsukuba $32 \mathrm{~m}$ antennas. The signal from LNA output is transferred to the observation room through optical fibers and sampled without any frequency conversions. The signals from LNA of the X-band and the S-band are combined with the remaining RF frequency.

KOMB [B]]. After the BWS, a $512 \mathrm{MHz}$ band became three times wider (about $1.5 \mathrm{GHz}$ wide). The S-band signal was used for the ionosphere correction by following the conventional way.

Finally we performed the baseline analysis with Calc/Solve [团] . The figure.⿴囗十 shows the baseline length between Kashima 11m and Tsukuba 32m stations from geodetic VLBI sessions in 2011. The result of last session is consistent with other results of conventional VLBI technique observations.

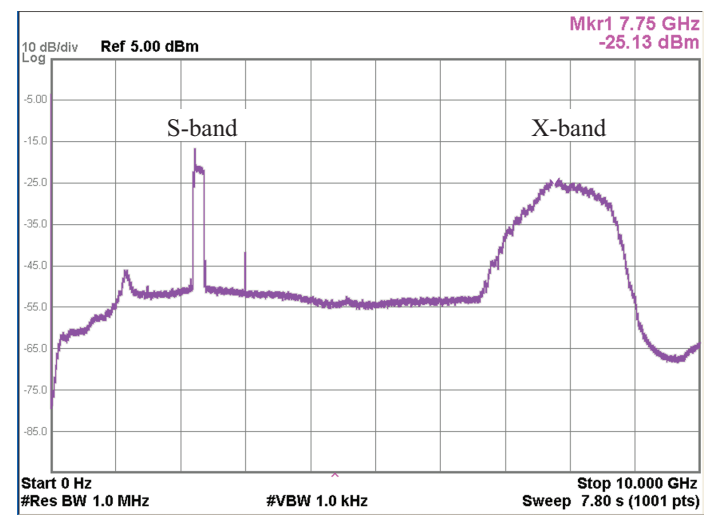

Figure 3: Spectrum of combined the S-band and the X-band with DSAMS technique by a spectrum analyzer 


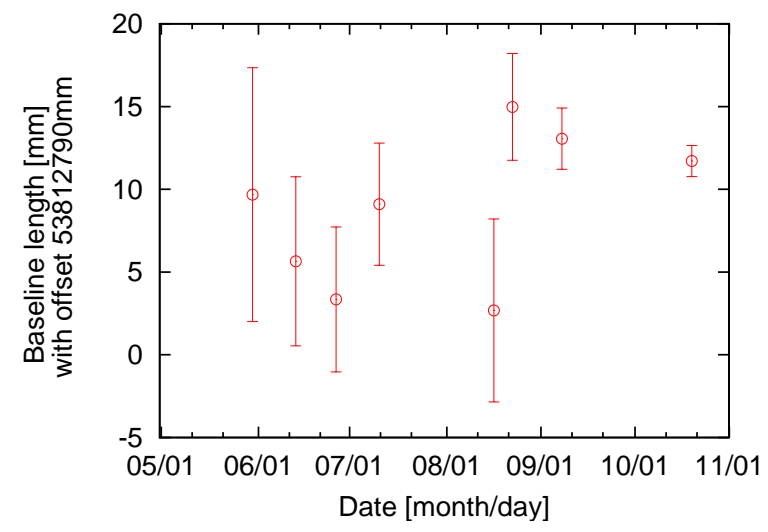

Figure 4: Baseline result between Kashima $11 \mathrm{~m}$ and Tsukuba $32 \mathrm{~m}$ stations in 2011. The DSAMS session was carried out in October 20, 2011 and other results were extracted from IVS database http://lupus.gsfc.nasa.gov/sess/master11.html.

\section{Gala-V, the next generation broadband VLBI system}

For VLBI-based frequency comparison, we are developing a new wideband VLBI system named GALA-V which has similar specifications as the next generation wideband VLBI observation system VLBI2010. Our system has fixed frequency bands at 3.2-4.2 GHz, 4.8-5.8 GHz, 9.6-10.6 GHz, and 12.8-13.8 GHz. The frequency arrangement was decided by taking into account the delay measurement performance, the radio interference conditions based on RFI field surveys, and the use of the RF direct sampling technique. All of these design choices contribute to a cost reduction for the system.

The figure.[1] shows the transportable 1.6 meter radio telescope which has been designed to be easily assembled/disassembled. The GALA-V system will include a pair of these small antennas and medium size antenna. The small antennas can be placed at frequency standard laboratories and the addition of medium size antenna will enable VLBI observations together with the small antennas by improving the signal to noise ratio, as the sensitivity of a VLBI system is proportional to the product of two antenna diameters. The anticipated precision on the delay measurements in combination with a $34 \mathrm{~m}$ or a $11 \mathrm{~m}$ telescope is between 6 and $8 \mathrm{ps}$ for a single observation with an integration time between 7 and $40 \mathrm{~s}$. Test observations with this prototype system will start in 2013.

\section{References}

[1] K. Takefuji, T. Kondo, M Sekido, T. Kumazawa,K. Harada, T. Nakayama,S. Kurihara,K. Kokado,R. Kawabata, and R. Ichikawa, "High-order Sampling Techniques of Aliased Signals for Very Long Baseline Interferometry," in Publications of the Astronomical Society of the Pacific, 2013, vol 124, issue 920, p.1105,

[2] M. Kimura, "Development of the software correlator for the vera sys-tem iii," Serial No. 29 October 2008, p. 12, 2008. 

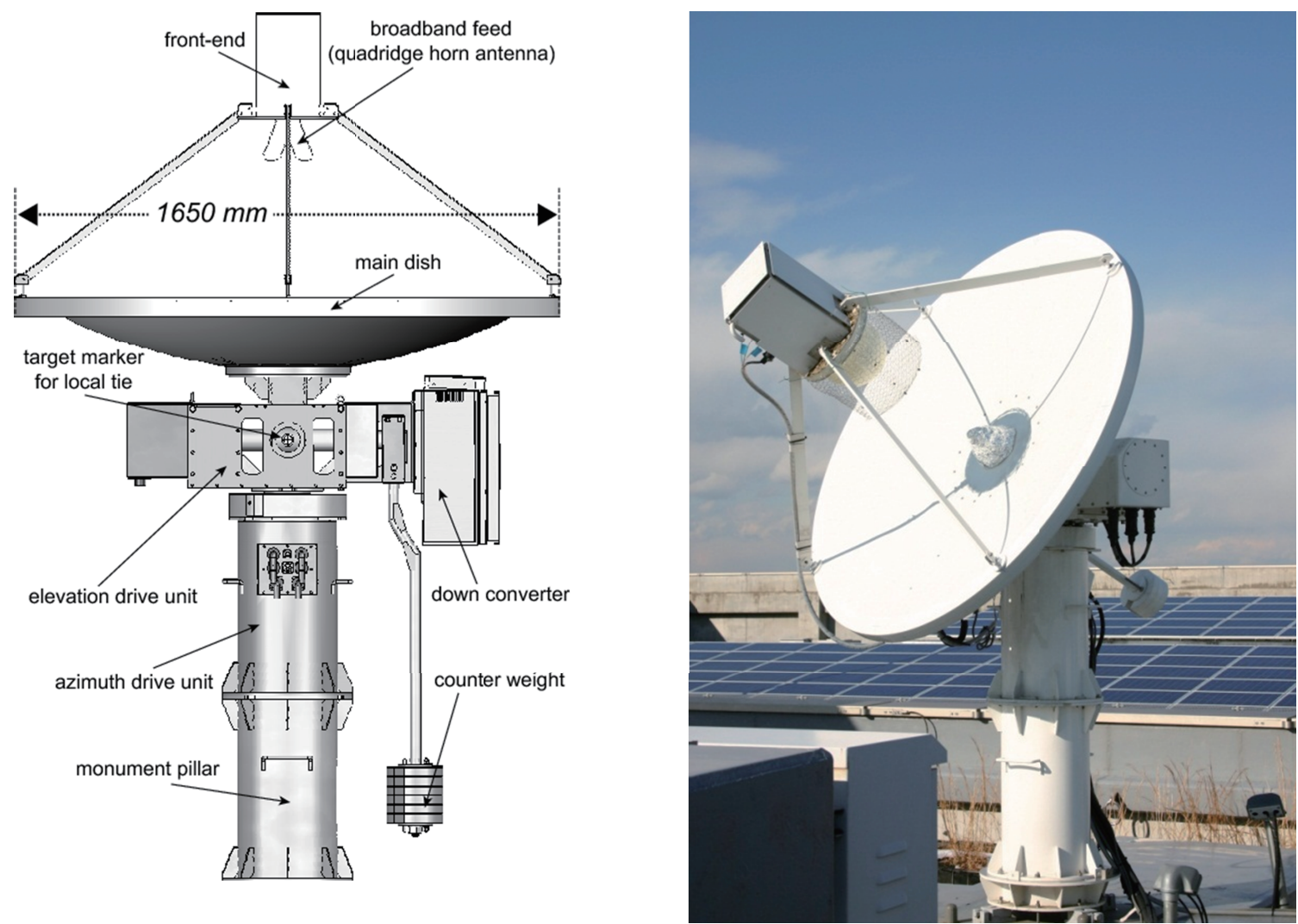

Figure 5: the transportable 1.6 meter radio telescope which has been designed to be easily assembled/disassembled. The receiver consists in a Quad-ridged horn antenna and a broadband LNA. The two linear polarized signals are transfered by optical cables and are sampled without any frequency conversions.

[3] T. Kondo, M. Sekido, and H. Kiuchi, "Ksp bandwidth synthesizing software," J. Comm. Res. Lab, vol. 46, no. 1, pp. 67-76, 1998.

[4] L. Petrov, "Mark-5 vlbi analysis software calc/solve," Web document http://gemini. gsfc. nasa. gov/solve, vol. 7, 2008. 